\title{
Análisis de la Literatura sobre Dispositivos Móviles en la Universidad Española
}

\section{Literature Review on Mobile Devices in Spanish University}

\author{
Julio César Mateus \\ Universitat Pompeu Fabra (España) \\ Sue Aran-Ramspott \\ Universitat Ramon Llull (España) \\ Maria-Jose Masanet \\ Universitat Pompeu Fabra (España)
}

\section{Resumen}

Este artículo presenta un análisis sistematizado de la producción científica entre los años 2012 y 2016 sobre el uso y aplicación de dispositivos móviles en el ámbito universitario español. El objetivo es analizar el interés académico por el Aprendizaje Móvil y las características de esta producción. Para ello se seleccionaron 50 artículos de revistas y comunicaciones de actas de congresos internacionales indexados en las bases de datos Web of Science, Scopus y Google Scholar y posteriormente se analizaron en torno a cuatro categorías: información sobre los autores, información bibliográfica de las publicaciones, información sobre las poblaciones estudiadas; e información científico-metodológica. Los resultados indican que se trata de un tema de desarrollo incipiente, dado el limitado número de artículos y comunicaciones halladas, pero esta producción evidencia la importancia de los grupos de investigación y los proyectos financiados, que permiten definir una trayectoria de investigación en varios autores. Asimismo, algunas comunidades autónomas españolas están subrepresentadas, así como algunas disciplinas abordadas en los estudios. En lo que respecta a la metodología, aparece como predominante la cuantitativa y como técnica de investigación más frecuente el cuestionario, aunque también se reconoce un número creciente de diseños experimentales. Las evidencias encontradas proponen un amplio campo para la investigación sobre el potencial pedagógico de los dispositivos móviles en la educación superior y permiten proponer una agenda para futuros estudios.

Palabras clave: tecnología de la educación; universidad; enseñanza y formación; aprendizaje móvil; dispositivos móviles. 


\begin{abstract}
This article presents a systematized review of scientific production between the years 2012 and 2016 of the use and application of mobile devices in Spanish universities. The objective is to interpret academic interest in Mobile Learning and the attributes of this production. In order to do this, 50 articles from journals and minutes from international congresses indexed in the databases of Web of Science, Scopus and Google Scholar were selected and subsequently analyzed within four categories: information about the authors, the publications' bibliographic information, information about the populations studied and scientific-methodological information. The results indicate that this is an emerging topic of development, given the limited number of articles and communications found, yet this output demonstrates the importance of the research groups and projects funded, which have defined a research trajectory in several authors. Likewise, some Spanish autonomous regions and certain disciplines addressed in the studies are underrepresented. The predominant methodology is quantitative and the most frequent research technique is the questionnaire, although there is also a growing number of experimental designs. The evidence found suggests a broad field for research into the pedagogical potential of mobile devices in higher education and puts forward an agenda for future studies.
\end{abstract}

Keywords: information and communication technologies; educational technology; university; teaching and training; mobile learning; mobile devices.

El interés académico por el Aprendizaje Móvil ha crecido desde inicios de siglo con la penetración acelerada de la tecnología móvil en la población. Aunque es difícil hallar el origen del término Mobile Learning o m-learning, Quinn lo definió en el año 2000 como una extensión del e-learning a partir de dispositivos móviles (citado en Berge y Muilenburg, 2013). Con el tiempo, el concepto fue encontrando desarrollos más complejos, interesados en una pedagogía centrada en el estudiante y en el aprovechamiento de las propiedades intrínsecas de los dispositivos en cuestión: portabilidad, conectividad, adaptabilidad e interactividad.

Entre otros planteamientos teóricos destacan el de los Entornos Personales de Aprendizaje (Castañeda y Adell, 2013) y el conectivismo (Siemens, 2005), que evidencian los cambios en el panorama educativo. Como advertía Pisanty (2009), "las tecnologías dan forma a las actividades, bien por facilitarlas o bien por limitarlas, y por otra parte las actividades y las comunidades fuerzan la creación de nueva tecnología y acompañando a ésta nuevos paradigmas". En este contexto, es apropiado preguntarse qué actividades e innovaciones se están llevando a cabo en el sistema educativo a partir de la penetración de los dispositivos móviles en la sociedad. Este trabajo tiene el objetivo de profundizar en esta área de conocimiento a través de una revisión sistematizada de la producción científica sobre la presencia y aplicación de dispositivos móviles en el entorno universitario en el ámbito español. 


\section{Dispositivos móviles en la educación superior: Estado de la cuestión}

Existen algunos consensos en relación a la aportación de los dispositivos móviles a la educación. En primer lugar, para garantizar el máximo efecto positivo deben interrelacionarse tres elementos: los componentes tecnológicos (software y hardware); el sentido y el contexto educativo en el que estos componentes actúan (diseño de experiencias de enseñanza aprendizaje relevantes y pertinentes); y los usuarios (docentes y discentes). Los esfuerzos deben orientarse, por tanto, a la armonización de estos elementos interdependientes.

Sung, Chang y Liu (2016) dieron cuenta del impacto positivo general que causa el uso de dispositivos móviles en contextos educativos en todos los niveles. Algunas de las ventajas frecuentes reportadas son la función de retroalimentación instantánea, que favorece la evaluación formativa y la gestión de grupos grandes, así como la función comunicativa que facilita el trabajo cooperativo de estudiantes y a la atención individual del aprendizaje. Otras tendencias identificadas son que el efecto de los dispositivos móviles en el aula mejora con la portabilidad; que son más eficaces cuando se utilizan para la indagación; que también lo son más en entornos informales que formales; $y$ que funcionan mejor cuando responden a intervenciones cortas o de duración media antes que a tareas de largo plazo. Asimismo, concluyeron que uno de los problemas recurrentes en la implementación de experiencias educativas con dispositivos móviles es la insuficiente preparación de los docentes.

Por su parte, Pimmer, Mateescu y Grohbiel (2016) concluyeron que la aplicación del aprendizaje móvil en entornos de educación superior es aún limitada porque los proyectos predominantemente adoptan un "enfoque instruccionista" (ligado al uso de dispositivos para presentar y evaluar contenidos). En cambio, cuando se diseñan proyectos "híbridos" (de enfoque construccionista, situado o colaborativo), las posibilidades transformadoras de los dispositivos móviles crecen notablemente.

Finalmente, Brazuelo y Gallego (2014) investigaron la producción científica española y advirtieron un interés creciente en relación a las nuevas funcionalidades de los dispositivos que se incorporan al ámbito educativo como los SMS, los podcast y los medios sociales, principalmente, pero también las apps o aplicaciones móviles, la realidad aumentada (RA) y los códigos de respuesta rápida $(\mathrm{QR})$.

\section{Marco referencial de la universidad española}

En España, el crecimiento de la tecnología móvil presenta indicadores de crecimiento en constante evolución. En la Unión Europea es solo superado por Suecia en cuanto a penetración de terminales digitales (Villalonga y Marta-Lazo, 2015). Así, "el 73,3\% de los hogares españoles contaba con conexión a Internet a finales de 2015 y el 76,1\% de los internautas utilizaba Internet diaria o casi diariamente" 
y "el teléfono móvil continúa consolidándose como principal dispositivo de acceso, utilizado por el 76,4\% de los internautas" (ONTSI, 2016, p. 255).

En el ámbito universitario también se observa una tendencia sostenida desde hace 10 años: "la docencia virtual, la gestión de licencias software para docencia y el soporte a aulas de informática de uso docente están implantados prácticamente en la totalidad de las universidades" (Píriz, 2015, p. 10). Del mismo modo, el 80\% de las aulas de docencia poseen, como mínimo, conexión a Internet y el 87\% de los estudiantes se conectan al menos una vez al año a la wifi de la universidad. La práctica totalidad del profesorado y de los estudiantes utilizan la plataforma de docencia virtual institucional y el número de titulaciones no presenciales ofertadas por las universidades españolas alcanza la cifra de 532, lo que supone alrededor del $8 \%$ de las titulaciones que ofertan. Igualmente, la mitad de las universidades han considerado, de forma aislada o planificada, iniciativas en relación con la adopción de cursos MOOC (Píriz, 2015, p. 11). En cuanto a las prácticas y usos, el 73,84\% del alumnado que cursa enseñanza superior prefiere los medios sociales para la docencia universitaria y el 6o,8\% emplea los chats frente a otras herramientas para su labor educativa (Vílchez, Reche y Marín, 2015).

\section{Tendencias en el Aprendizaje Móvil}

Durall et al. (2012) consideran que este tema resulta de especial interés para la docencia, el aprendizaje, la investigación y la gestión de la información por lo siguiente:

La rápida adopción de teléfonos inteligentes posibilita utilizar los dispositivos de los propios estudiantes y docentes como herramientas para la docencia y el aprendizaje.

El acceso a la información en cualquier momento y lugar permite una experiencia de aprendizaje flexible y personalizado, en la que el contexto adquiere importancia.

El desarrollo del m-learning implica la aparición de nuevos modelos y metodologías de presentación de los contenidos de aprendizaje.

Los móviles propician la aparición de nuevas dinámicas de interacción entre docentes y estudiantes, y favorecen el desarrollo de prácticas colaborativas.

El estudio prospectivo Universidad 2020: Papel de las TIC en el nuevo entorno socioeconómico, publicado el 2011, ya reclamaba un cambio profundo que asuma como realidades la conectividad total, en el marco del Internet de las Cosas (IoT); la aparición de nuevos y más potentes dispositivos móviles, que definen un nuevo tipo de usuario; y la puesta en red a través del cloud computing de todos los servicios de forma estándar e interoperable. A esto se suma el hecho de que los dispositivos móviles juegan un rol crucial para garantizar la inclusión en la medida en que permiten atender algunas discapacidades (visual, auditiva, motriz e intelectual) a través de diversas aplicaciones y funcionalidades (Fundación Telefónica, 2011). Ante esta realidad que, en igual medida entusiasma y preocupa, es imperativo conocer 
cómo la producción académica está abordando la presencia de los dispositivos móviles en la educación superior.

\section{METODOLOGÍA}

Se realizó una revisión sistematizada de literatura científica publicada entre los años 2012 al 2016, basada en casos o investigaciones realizadas en universidades españolas y disponibles en tres bases de datos de referencia. El corpus de estudio final, luego de aplicar los filtros de exclusión y depurar los resultados fue de 50 artículos (ver anexo). Los objetivos de esta revisión fueron (i) explorar cómo ha sido abordado el objeto de estudio y (ii) analizar las tendencias y brechas en la investigación, de modo que se puedan ofrecer algunas pistas para futuras investigaciones.

Los pasos seguidos para llevar a cabo la revisión sistematizada fueron: (i) definición de los conceptos de búsqueda, (ii) definición del universo de la búsqueda, (iii) aplicación de criterios de inclusión y exclusión, (iv) definición de variables de análisis y estandarización, y (v) gestión de los datos y análisis de los resultados.

Para complementar la revisión se realizó un análisis bibliométrico, que se caracteriza por una revisión sistemática de fuentes definidas en un período de tiempo con el objetivo de localizar, analizar, ordenar, contar y evaluar la presencia de los datos o secuencias de la búsqueda. Este tipo de análisis sistematizado permite que el proceso sea científico y transparente y que pueda ser replicado (Tranfield, Denyer y Smart, 2003).

\section{Paso 1- Definición de los conceptos de búsqueda}

Dada la variedad y dispersión de palabras clave utilizadas en la literatura científica, se trabajó con una búsqueda combinada, aplicando conectores booleanos y parámetros estandarizados. Se utilizó la siguiente ecuación de búsqueda en inglés aplicada a los metadatos (títulos, resúmenes y palabras clave), por ser la que mejores resultados generó: "mobile learning" OR "mobile devices" OR "digital devices" OR smartphones OR tablets AND university OR "higher education" AND "Spain". La búsqueda se realizó únicamente en inglés, pues es el idioma exigido por todas las revistas para la inclusión de los metadatos. Fueron seleccionados, sin embargo, textos publicados en inglés o en castellano.

\section{Paso 2 - Definición del universo de la búsqueda}

En esta fase se procedió a explorar artículos publicados entre el 2012 y el 2016 en revistas científicas y comunicaciones publicadas en actas de congresos internacionales indexadas en las bases de datos y buscadores Web of Science (WoS) y Scopus. Luego se amplió la búsqueda a Google Scholar. Delgado y Repiso (2013) aconsejan el uso de 
Google Scholar por cuanto "no solo cubre muchas más revistas que sus competidores (WoS y Scopus) sino que carece del sesgo anglosajón que poseen estos, pues acoge revistas procedentes de más países y escritos en más lenguas" (p. 50).

\section{Paso 3 - Aplicación de criterios de inclusión y exclusión}

A los criterios establecidos en los primeros pasos, se añadieron los siguientes criterios de inclusión y exclusión para la depuración del corpus de estudio:

Criterios para incluir el artículo o comunicación: (i) aborda de forma explícita el uso de dispositivos móviles (tablets y smartphones); (ii) su aplicación o campo de referencia es alguna universidad española; (iii) incluye -aunque no se limite- el ámbito educativo presencial; y (iv) es accesible en su totalidad desde la base de datos.

Criterios para excluir: (i) fue publicado antes del año 2012; (ii) no menciona de forma explícita el lugar donde realiza el estudio ni ubica a España como contexto referencial; (iii) no se circunscribe al ámbito universitario; (iv) se centra en educación a distancia; (v) no está escrito en idioma inglés o castellano; y (vi) solo se dispone del resumen pero no del texto completo.

\section{Paso 4- Definición de variables de análisis y estandarización}

Con el propósito de establecer un análisis sistematizado se utilizaron las siguientes variables para el análisis, las mismas que fueron estandarizadas y permitieron la formulación de preguntas de investigación, organizadas en 4 categorías:

Tabla 1. Estandarización de las variables de análisis y preguntas de investigación

\begin{tabular}{|l|l|l|}
\hline \multicolumn{1}{|c|}{ Variable } & \multicolumn{1}{c|}{ Estandarización } & \multicolumn{1}{c|}{ Pregunta } \\
\hline 1.Información genérica sobre los autores e instituciones \\
\hline $\begin{array}{l}\text { Número y género } \\
\text { de investigadores }\end{array}$ & $\begin{array}{l}\text { Firma normalizada de los } \\
\text { autores y precisión del género } \\
\text { (Masculino/Femenino) }\end{array}$ & $\begin{array}{l}\text { P1.1 ¿Quiénes son los investigadores } \\
\text { con mayor número de } \\
\text { publicaciones? ¿Qué género tienen? }\end{array}$ \\
\hline $\begin{array}{l}\text { Filiación } \\
\text { institucional }\end{array}$ & $\begin{array}{l}\text { Señalada en el artículo por cada } \\
\text { autor }\end{array}$ & $\begin{array}{l}\text { P1.2 ¿De qué universidades } \\
\text { proceden los investigadores? }\end{array}$ \\
\hline 2. Información bibliográfica de las publicaciones \\
\hline $\begin{array}{l}\text { Año de } \\
\text { publicación }\end{array}$ & $\begin{array}{l}\text { Año de publicación del artículo } \\
\text { (2012-2016) }\end{array}$ & $\begin{array}{l}\text { P2.1 ¿Existe alguna variación } \\
\text { respecto a la producción de cada } \\
\text { uno de los años analizados? }\end{array}$ \\
\hline Palabras clave & $\begin{array}{l}\text { Señaladas por el autor en el } \\
\text { idioma principal del artículo } \\
\text { o comunicación (número } \\
\text { indeterminado) }\end{array}$ & $\begin{array}{l}\text { P2.2 ¿Qué palabras clave utilizan y } \\
\text { cuáles son las más relevantes? }\end{array}$ \\
\hline
\end{tabular}




\begin{tabular}{|c|c|c|}
\hline Variable & Estandarización & Pregunta \\
\hline $\begin{array}{l}\text { Tipo de } \\
\text { publicación }\end{array}$ & $\begin{array}{l}\text { Artículo (publicada en alguna } \\
\text { revista indexada peer-review) o } \\
\text { comunicación en algún congreso } \\
\text { científico (publicada en un Acta o } \\
\text { Libro) }\end{array}$ & \multirow[t]{2}{*}{$\begin{array}{l}\text { P2.3 ¿Dónde se publicaron las } \\
\text { investigaciones y en qué lengua? }\end{array}$} \\
\hline Lengua & $\begin{array}{l}\text { Idioma del artículo o } \\
\text { comunicación (inglés o } \\
\text { castellano) }\end{array}$ & \\
\hline \multicolumn{3}{|c|}{ 3. Información sobre las poblaciones estudiadas } \\
\hline Lugar & $\begin{array}{l}\text { Ámbito geográfico donde se } \\
\text { realizó la investigación (en caso } \\
\text { de ser empírica). }\end{array}$ & $\begin{array}{l}\text { P3.1 ¿Cuáles han sido las } \\
\text { poblaciones objeto de estudio? } \\
\text { ¿Hay comunidades autónomas } \\
\text { subrepresentadas en los estudios? }\end{array}$ \\
\hline Disciplina & $\begin{array}{l}\text { Precisar, si corresponde, la } \\
\text { disciplina a la que pertenece la } \\
\text { muestra. }\end{array}$ & \multirow{2}{*}{$\begin{array}{l}\text { P3.2 ¿En qué disciplinas se ha } \\
\text { puesto mayor atención? ¿Se ha } \\
\text { trabajado más con estudiantes de } \\
\text { grado o posgrado? }\end{array}$} \\
\hline Grado o Posgrado & $\begin{array}{l}\text { Precisar, si corresponde, si la } \\
\text { muestra con la que se trabajó en } \\
\text { el estudio de campo es de grado } \\
\text { o posgrado (grado, posgrado, no } \\
\text { precisa) }\end{array}$ & \\
\hline \multicolumn{3}{|c|}{ 4. Información científico-metodológica de las publicaciones } \\
\hline $\begin{array}{l}\text { Abordaje } \\
\text { científico }\end{array}$ & $\begin{array}{l}\text { Precisar si se trata de un artículo } \\
\text { teórico-ensayístico o presenta } \\
\text { una investigación de carácter } \\
\text { empírico (define objetivo, método } \\
\text { y resultados). }\end{array}$ & $\begin{array}{l}\text { P4.1 ¿Qué tipo de investigación se } \\
\text { realiza: teórica o empírica? }\end{array}$ \\
\hline Metodología & Cuantitativa / Cualitativa / Mixta & $\begin{array}{l}\text { P4.2 ¿Qué metodologías son más } \\
\text { utilizadas? }\end{array}$ \\
\hline $\begin{array}{l}\text { Técnica de } \\
\text { investigación }\end{array}$ & $\begin{array}{l}\text { Precisar la técnica utilizada } \\
\text { (análisis de contenido, } \\
\text { cuestionario, entrevista, } \\
\text { etnografía, etc. o si no procede) }\end{array}$ & $\begin{array}{l}\text { P4.3 ¿Qué técnicas e instrumentos } \\
\text { son más utilizados y cuáles menos? }\end{array}$ \\
\hline Dispositivo & $\begin{array}{l}\text { Precisar tablet o smartphone u } \\
\text { otro }\end{array}$ & $\begin{array}{l}\text { P4.4 ¿A qué dispositivo han } \\
\text { prestado mayor atención las } \\
\text { investigaciones? }\end{array}$ \\
\hline
\end{tabular}

Elaboración propia 


\section{Paso 5- Gestión de los datos y análisis de los resultados}

Para la gestión de las referencias se utilizó el software Mendeley y el análisis fue elaborado en una tabla manual en Excel. Se ha realizado un análisis descriptivo univariante.

\section{RESULTADOS}

A continuación presentamos los resultados obtenidos a partir de la revisión y análisis de los 50 artículos seleccionados como corpus de estudio (ver anexo). Están organizados en cuatro secciones: Información genérica sobre los autores e instituciones; información bibliográfica de las publicaciones; información sobre las poblaciones estudiadas; e información científico-metodológica de las publicaciones.

\section{Información genérica sobre los autores e instituciones}

P1.1 ¿Quiénes son los investigadores con mayor número de publicaciones? ¿Qué género tienen los investigadores?

En total, se encontraron 111 autores firmantes de las publicaciones. El 54,1\% son hombres y 45,9\% mujeres. En promedio, cada publicación fue firmada por 2,6 investigadores. Los autores con mayor producción de la muestra analizada son:

Tabla 2. Investigadores con mayor producción científica sobre el tema

\begin{tabular}{|l|l|l|l|}
\hline \multicolumn{1}{|c|}{ Autor } & \multicolumn{1}{|c|}{ Sexo } & \multicolumn{1}{c|}{ Institución } & Publicaciones \\
\hline Vázquez-Cano, E. & Masculino & $\begin{array}{l}\text { Universidad Nacional de Educación } \\
\text { a Distancia }\end{array}$ & 5 \\
\hline Sevillano-García, M. L. & Femenino & $\begin{array}{l}\text { Universidad Nacional de Educación } \\
\text { a Distancia }\end{array}$ & 5 \\
\hline Pascual-Sevillano, M. A. & Femenino & Universidad de Oviedo & 3 \\
\hline Fombona, J. & Masculino & Universidad de Oviedo & 3 \\
\hline García-Peñalvo, F. J. & Masculino & Universidad de Salamanca & 3 \\
\hline
\end{tabular}

Elaboración propia

Es importante señalar que los primeros cuatro autores con mayor producción son parte de un proyecto de investigación común, denominado "Aprendizaje ubicuo con dispositivos móviles: elaboración y desarrollo de un mapa de competencias en educación superior” y financiado por el Ministerio de Ciencia e Innovación de España. Asimismo, han realizado otras investigaciones en áreas de interés vinculadas. 
En el caso del autor Francisco García-Peñalvo, se trata de un investigador con un importante número de publicaciones sobre este tema.

\section{P1.2 ¿De qué universidades proceden los investigadores?}

Las universidades afiliadas a los autores de las publicaciones analizadas suman 40, de ellas 35 son españolas y cinco extranjeras. En cuanto a las que tienen mayor número de publicaciones están la Universidad Nacional de Educación a Distancia, con 11 publicaciones; seguida de las universidades de La Laguna y Oviedo, con cuatro cada una; y las de Salamanca, Islas Baleares, y Politécnica de Valencia, con tres.

Tabla 3. Universidades con mayor producción científica sobre el tema

\begin{tabular}{|l|l|}
\hline \multicolumn{1}{|c|}{ Universidad } & \multicolumn{1}{c|}{ Publicaciones } \\
\hline Universidad Nacional de Educación a Distancia & 11 \\
\hline Universidad de La Laguna & 4 \\
\hline Universidad de Oviedo & 4 \\
\hline Universidad de Salamanca & 3 \\
\hline Universitat de les Illes Balears & 3 \\
\hline UniversitatPolitècnica de València & 3 \\
\hline
\end{tabular}

Elaboración propia

\section{Información bibliográfica de las publicaciones}

P2.1 ¿Existe alguna variación respecto a la producción de cada uno de los años analizados?

La producción científica se incrementa anualmente, salvo en el caso del 2016. Así, el 2012 se hallaron tres publicaciones; el 2013, nueve, el 2014, 14; el 2015, 16 y en el 2016 la cifra desciende a ocho publicaciones. Estos datos evidencian que se trata de un tema de interés en el campo educativo, ya que las investigaciones aumentaron anualmente, salvo el 2016. El año en curso, 2017, quedará confirmar si se trata de una tendencia a la baja e indagar en las razones. 
Figura 1. Número de publicaciones anuales

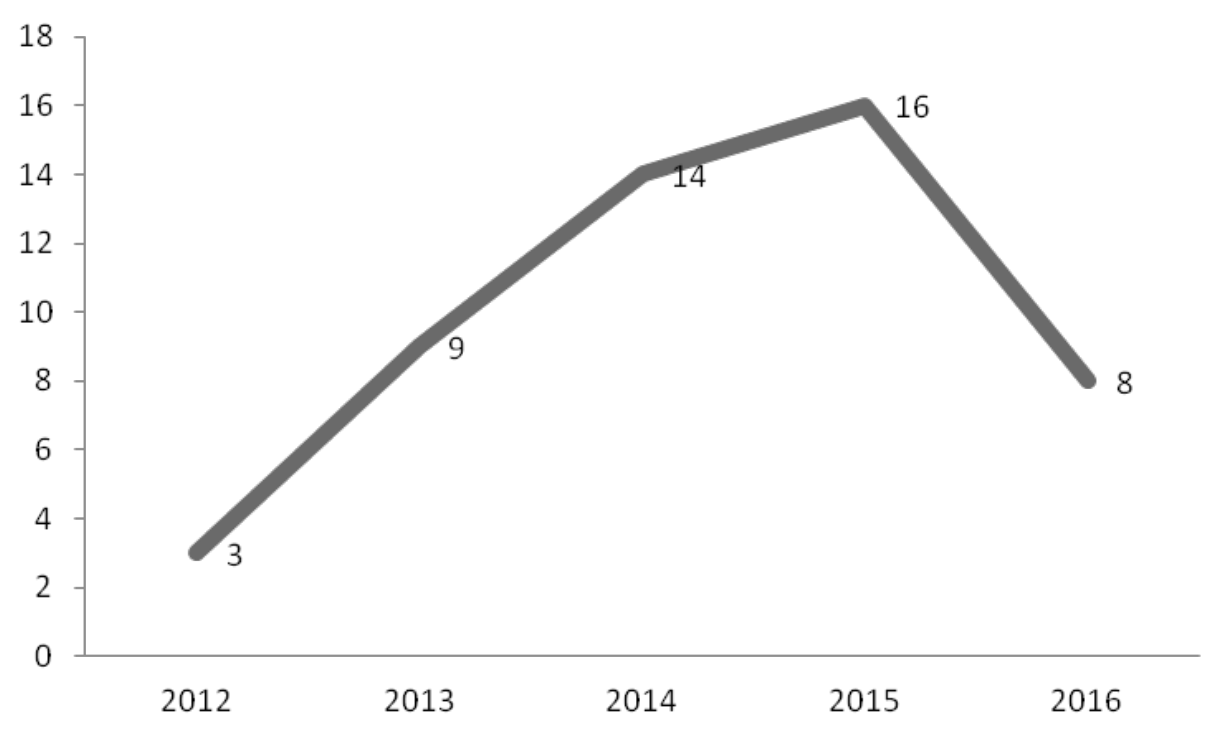

Elaboración propia

\section{P2.2 ¿Qué palabras clave utilizan y cuáles son las más relevantes?}

En total, se identificaron 131 palabras clave. Las más repetidas fueron higher education (20 menciones), mobile learning (10 menciones), smartphones (nueve menciones), ICT (ocho repeticiones) y mobile devices (ocho menciones). En la siguiente tabla se observa la dispersión de palabras clave, lo que genera dificultad para encontrarlas en las bases de datos, y el uso de palabras clave no normalizadas que corresponden al mismo ámbito (e.g. "mlearning" en lugar de "m-learning"; el uso indistinto de formas singulares o plurales e.g. "tablet" y "tablets"; o de modelos específicos e.g. "iPad" en vez del nombre del dispositivo "tablet"). En muchas publicaciones no se exige el uso de un tesauro o lista de palabras clave, por lo que cada autor optar por colocar las que considera pertinentes. 
Tabla 4. Combinaciones de palabras clave más usadas

\begin{tabular}{|l|l|l|l|}
\multicolumn{1}{|c|}{$\begin{array}{c}\text { Palabra más } \\
\text { repetida }\end{array}$} & \multicolumn{1}{|c|}{ Alternativa 2 } & \multicolumn{1}{c|}{ Alternativa 3 } & \multicolumn{1}{c|}{ Alternativa 4 } \\
\hline higher education (20) & university student (2) & EHEA (2) & -- \\
\hline mobile learning (10) & m-learning (3) & mlearning (2) & $\begin{array}{l}\text { mobile and } \\
\text { ubiquitouslearning } \\
\text { (1) }\end{array}$ \\
\hline smartphones (9) & -- & -- & -- \\
\hline ICT (8) & technology (4) & new technologies (3) & $\begin{array}{l}\text { ICT applied to } \\
\text { education (1) }\end{array}$ \\
\hline mobile devices (8) & $\begin{array}{l}\text { digital mobile devices } \\
(2)\end{array}$ & digital devices (1) & -- \\
\hline tablets (4) & tablet pc (3) & iPad (2) & digital tablet (2) \\
\hline ubiquity (4) & ubiquitous (2) & $\begin{array}{l}\text { ubiquitous learning } \\
(2)\end{array}$ & -- \\
\hline apps (4) & educational apps (1) & $\begin{array}{l}\text { multimedia } \\
\text { applications (1) }\end{array}$ & -- \\
\hline electronic book (2) & e-books (1) & e-book (1) & -- \\
\hline
\end{tabular}

Elaboración propia

\section{P2.3 ¿Dónde se publicaron las investigaciones y en qué lengua?}

De las 50 publicaciones seleccionadas, 42 fueron artículos de revistas indexadas. Las ocho restantes fueron publicadas en actas de congresos internacionales. Del total de revistas (42), 27 son españolas y 15 son editadas en otros países (Alemania, Brasil, Colombia, Chile, EEUU, Grecia, México, Nueva Zelanda, Reino Unido y Turquía). Entre las revistas españolas que publicaron el mayor número de artículos analizados destacan Historia y Comunicación Social (cinco artículos), Revista de Universidad y Sociedad del Conocimiento (tres artículos) y Comunicar y Revista Complutense de Educación (dos artículos cada una). En su gran mayoría, los tópicos centrales de las revistas donde se publicaron fueron educación (20 veces, incluyendo tecnología educativa como tema vinculado) y en menor medida comunicación (ocho veces), estudios interdisciplinarios ( 5 veces) y otros (medicina, geografía, metodologías en ciencias sociales y psicología, etc.).

En cuanto a la lengua, 32 del total de publicaciones fueron publicadas en castellano y 18 en inglés. Con excepción de una sola comunicación, el resto de comunicaciones fueron publicadas en inglés, lo que confirma la tendencia internacional del uso de este idioma como lingua franca de la comunicación científica. 
Figura 2. Fuentes y lenguas de las publicaciones

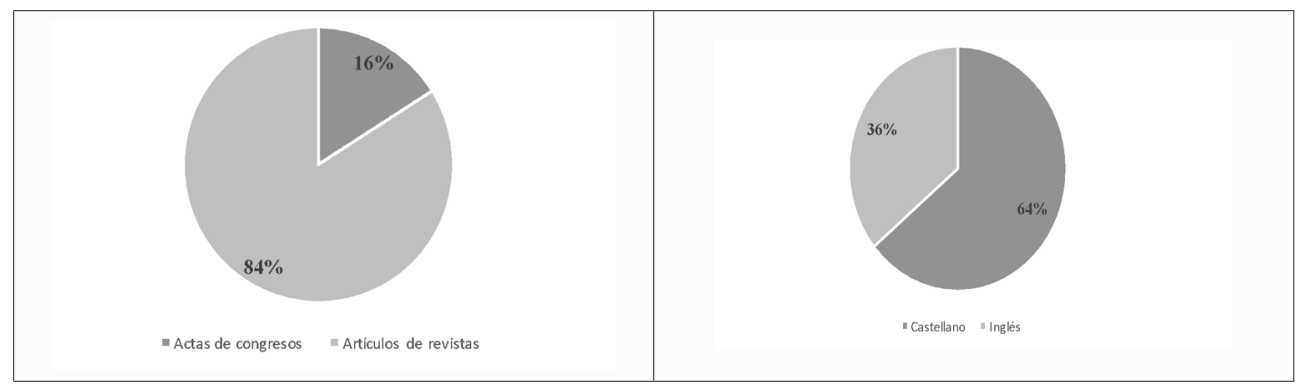

Elaboración propia

\section{Información sobre las poblaciones estudiadas}

\section{P3.1 ¿Cuáles han sido las poblaciones objeto de estudio? ¿Hay comunidades autónomas subrepresentadas en los estudios?}

La revisión efectuada de la literatura científica (2012-2016) basada en investigaciones realizadas en universidades españolas permite observar si hay publicaciones sobre el objeto de estudio referidas a todas las comunidades autónomas o bien hay una presencia desigual como población objeto de análisis. Los resultados indican cuáles son las comunidades más estudiadas y cómo cinco no aparecen como objeto de análisis en las investigaciones publicadas. Entre las comunidades autónomas que han sido objeto de análisis en mayor número de publicaciones destaca manifiestamente la Comunidad de Madrid, con 11 referencias que prácticamente duplican en número a las comunidades que la siguen en segundo lugar, Andalucía y Castilla-León, ambas con 6 publicaciones. En cuarto lugar estaría el Principado de Asturias (cinco publicaciones), seguido por la Comunidad Valenciana (cuatro publicaciones). Por debajo de las cuatro publicaciones se sitúan el resto de las comunidades. No hay ninguna referencia a investigaciones que hayan puesto bajo el foco a las comunidades de Aragón, Castilla-La Mancha, Ceuta, La Rioja o Melilla.

Es importante señalar que una misma investigación puede incluir más de una población, por lo que el número total de menciones de las comunidades autónomas que se observa en la tabla 6 es superior al total de investigaciones consultadas.

Por otro lado, no se han contemplado en esta tabla las publicaciones originadas desde universidades a distancia, dado que no explicitan las poblaciones de estudio (Universidad Nacional de Educación a Distancia, con cuatro publicaciones; Universitat Oberta de Catalunya, con una; y Universidad Abierta de Lisboa, junto con la Universidad de Oviedo, con otra). Tampoco se han recogido aquí los artículos 
de orientación estrictamente teórica (cuatro publicaciones) dado que tampoco se han referido a una población o comunidad concreta.

Los resultados indican que, efectivamente, hay comunidades autónomas subrepresentadas en los estudios. Destaca la representación mayoritaria de la Comunidad de Madrid, encabezado por Madrid capital, junto con las de Andalucía y Castilla-León. Sorprende que algunas comunidades con demografía importante y con una amplia red de universidades estén poco presentes como objeto de estudio (en particular, los casos de Cataluña y País Vasco).

Tabla 5. Comunidades Autónomas sobre las que se han realizado las investigaciones

\begin{tabular}{|l|l|}
\hline \multicolumn{1}{|c|}{ Comunidad Autónoma } & Publicaciones \\
\hline Comunidad de Madrid (Madrid 10, Alcalá 1) & 11 \\
\hline Andalucía (Córdoba 1, Granada 4, Sevilla 1) & 6 \\
\hline Castilla y León (Salamanca 4, Valladolid 1) & 5 \\
\hline Principado de Asturias (Oviedo 5) & 5 \\
\hline Comunidad Valenciana (Alicante 1+ Elche 1-, Valencia 2) & 4 \\
\hline Cataluña (Barcelona 1, No concreta 2) & 3 \\
\hline Galicia (Vigo 3) & 3 \\
\hline Islas Baleares (3) & 3 \\
\hline Islas Canarias (Santa Cruz de Tenerife -Tenerife 3) & 3 \\
\hline Región de Murcia (Cartagena 3) & 3 \\
\hline Cantabria (Santander 2) & 2 \\
\hline Extremadura (Badajoz-Mérida 1) & 1 \\
\hline Navarra (1) & 1 \\
\hline País Vasco (Bilbao 1) & 1 \\
\hline
\end{tabular}

Elaboración propia

P3.2 ¿En qué disciplinas se ha puesto mayor atención? ¿Se ha trabajado más con estudiantes de grado o posgrado?

Las publicaciones consultadas abordaron poblaciones variadas: hubo 28 menciones a disciplinas distintas y, en otros casos no contabilizados en este apartado, solo alusiones genéricas del tipo "estudiantes universitarios" o "estudios diversos de grado", sin precisar la especialidad.

Para organizar esta información, agrupamos las disciplinas en seis áreas de conocimiento genéricas. En primer lugar, con 25 menciones, las investigaciones se enfocan en áreas de Ingeniería y Arquitectura (que incluye carreras como Ingeniería Agrícola, Ingeniería Civil, Ingeniería de Sistemas, Ingeniería Industrial, Ingeniería Informática, Telecomunicación, Telemática y Tecnología de la Información y la 
Comunicación). En segunda posición, el área de Ciencias Sociales y Jurídicas, con 18 menciones (incluyendo Ciencias Sociales, Derecho, Ciencias de la Información, Comunicación, Comunicación Audiovisual, Filología, Geografía, Historia y Humanidades). En tercera posición, con 12 menciones, el área de Ciencias de la Salud (incluyendo Medicina, Odontología y Biología). Luego, en cuarto lugar, el área de Ciencias (que integran Ciencias Ambientales, Ciencia de Materiales, Matemática, Química, Nanociencia y Nanotecnología molecular). En quinto lugar está el área de Ciencias de la Educación, con 10 menciones (incluyendo las carreras de Pedagogía y Educación Social). Finalmente, en el sexto lugar, aparece el área de Artes y Humanidades, con cinco menciones.

Figura 3. Disciplinas de las poblaciones estudiadas

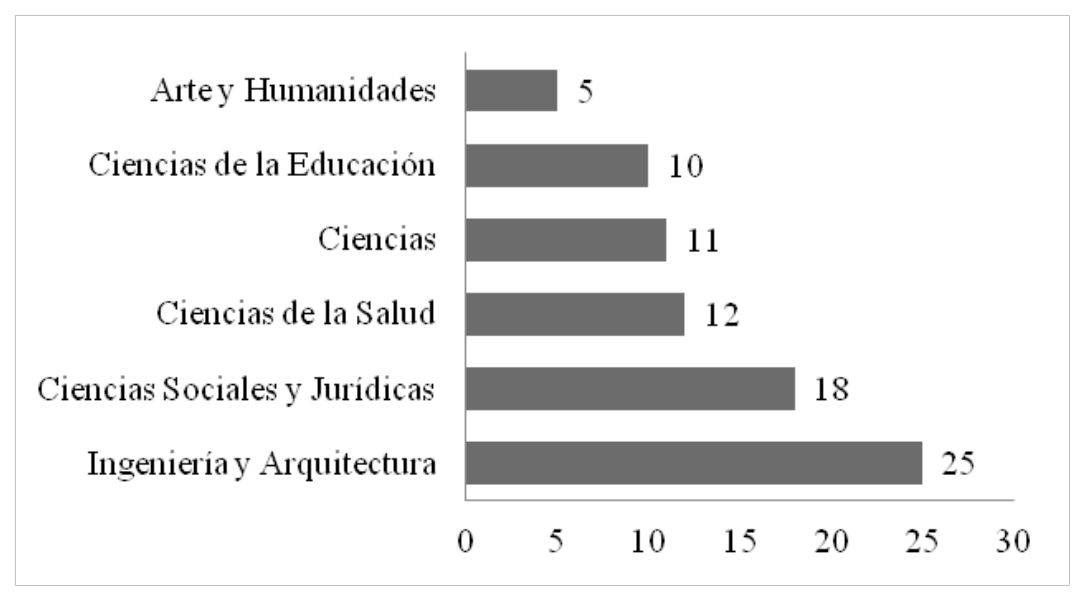

Elaboración propia

En cuanto al nivel de estudios, casi la totalidad de menciones (que representan el 94\% de la muestra) aborda estudios de grado y solo el $6 \%$ corresponde a posgrados (másteres, doctorados o cursos de posgrado en general).

\section{Información científico-metodológica de las publicaciones}

\section{P4.1 ¿Qué tipo de investigación se realiza: teórica o empírica?}

El tipo de investigación de 44 de las 50 publicaciones estudiadas es empírico, esto quiere decir que exhiben resultados a partir de un trabajo de campo. Las seis publicaciones restantes son de corte teórico, elaboradas a partir de la revisión documental de fuentes secundarias. En el caso de las investigaciones empíricas, 
destacan las validaciones de apps o software específico, usualmente en un grupo seleccionado de estudiantes de una o más universidades, y algunos experimentos donde se miden los efectos del aprendizaje manipulando el uso de determinadas aplicaciones o dispositivos.

Figura 4. Tipo de investigación

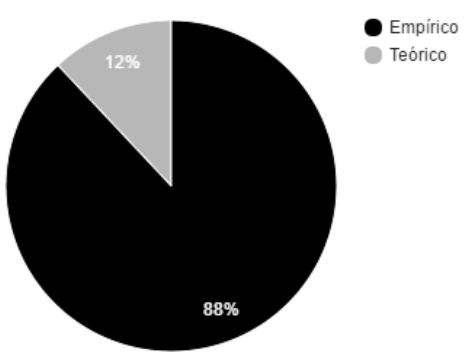

Elaboración propia

\section{P4.2 ¿Qué metodologías son más utilizadas?}

En cuanto a las metodologías aplicadas, destacan las cuantitativas, con 31 publicaciones, mientras que solo tres aplicaron una metodología cualitativa. Siete investigaciones fueron elaboradas a partir de una metodología mixta (cualitativa y cuantitativa). Llama la atención también que algunas de las publicaciones no señalen de forma explícita el tipo de metodología aplicada.

Figura 5. Metodologías aplicadas

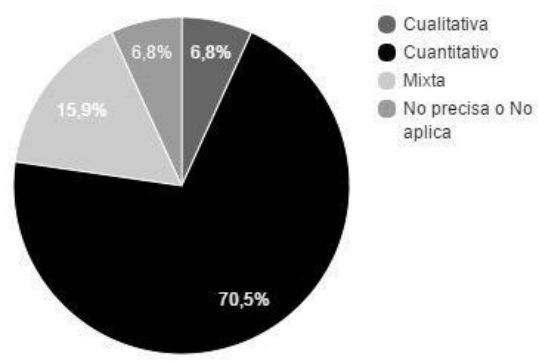

Elaboración propia 


\section{P4.3 ¿Qué técnicas e instrumentos son más utilizados y cuáles menos?}

La técnica cuantitativa por excelencia fue la encuesta a partir de la elaboración de cuestionarios (con preguntas cerradas o abiertas), mientras que en el caso de las investigaciones cualitativas (exclusivamente cualitativas o mixtas) se usaron técnicas y herramientas diversas, como las entrevistas en profundidad (3 menciones), la etnografía multisituada ( 2 menciones), los grupos focales ( 2 menciones), la observación, el análisis de contenido, el análisis de usabilidad, los grupos de discusión, las historias de vida y el método Delphi. Resulta interesante anotar que ocho de las publicaciones $(13,8 \%)$ utilizaron diseños experimentales y cuasiexperimentales con manipulación de variables (sobre todo de presencia/ausencia de dispositivos en contextos específicos).

Figura 6. Técnicas de investigación

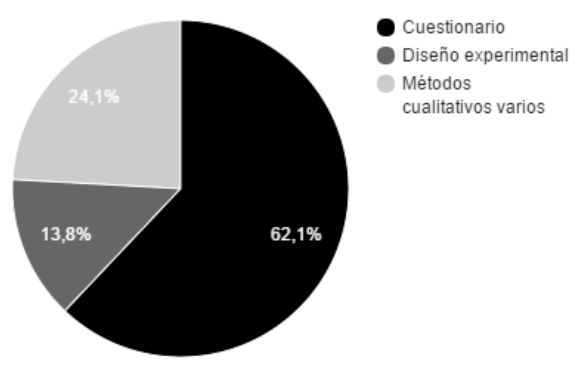

Elaboración propia

\section{P4.4 ¿A qué dispositivo han prestado mayor atención las investigaciones?}

La mayoría de publicaciones (31 de 50) no centran su atención en ningún dispositivo específico, sino que se refieren a los dispositivos de modo genérico, nombrando otros adicionales (e.g. ordenadores portátiles o laptops, phablets, clickers o Personal Remote System-PRS). Apenas ocho publicaciones centran su atención en los smartphones como único dispositivo y seis publicaciones solo en las tablets. Cinco trabajos hacen referencia a ambos dispositivos en la misma investigación.

También cabe resaltar el interés, además de los dispositivos, en el desarrollo de metodologías queprivilegien la participación (a través de experiencias departicipación directa con dispositivos móviles ad-hoc, como clickers, o aplicaciones que se pueden descargar en tablets y smartphones), en el uso de dispositivos para la evaluación (desarrollo de tests en línea o e-portafolios), la simulación (particularmente en el caso de las ciencias y las ingenierías), la adaptación de aplicaciones existentes (como Whatsapp), la creación de contenido para Realidad Aumentada a partir de códigos 
QR, o el desarrollo de estrategias didácticas más complejas que integran varios dispositivos. Otro elemento a destacar es el uso de ordenadores portátiles o laptops, que siguen vigentes en el desarrollo de aplicaciones y experiencias.

Figura 7. Dispositivos priorizados en las publicaciones

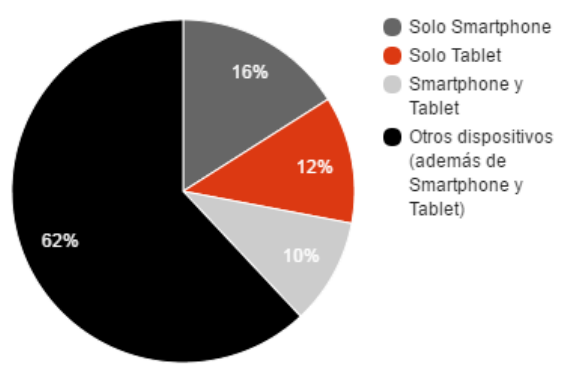

Elaboración propia

\section{CONCLUSIONES Y DISCUSIÓN}

De la información sobre los autores e instituciones se concluye que el número total de publicaciones hallado en las tres bases de datos consultadas es aún limitado. Aunque desde el 2012 el número de publicaciones se incrementó anualmente, el 2016 disminuyó por primera vez en el periodo estudiado, lo que nos invita a indagar si se trata de una tendencia y hacia dónde se dirige la agenda de investigación. Se concluye también que muchas de las publicaciones pertenecen a investigadores y grupos de investigación que forman parte de proyectos mayores (a veces con financiamiento, según se señala), lo que permite consolidar una trayectoria y una especialización a través de los años de ciertos autores. Asimismo, es notorio el interés de algunas universidades en el tema (especialmente la Universidad Nacional de Educación a Distancia). Son escasas las publicaciones individuales, lo que confirma la tendencia de la autoría múltiple en las publicaciones académicas.

A partir de la información bibliográfica, se aprecia una alta dispersión de palabras clave utilizadas. $\mathrm{Al}$ respecto, sugerimos utilizar un tesauro o un listado de palabras normalizadas para promover que aparezcan en los motores de búsqueda con más facilidad. Asimismo, el idioma inglés es cada vez más relevante para la comunicación científica, sobre todo para acceder a revistas indexadas de alto impacto y congresos internacionales.

En cuanto a las poblaciones presentes en las publicaciones, los resultados indican que, efectivamente, hay comunidades autónomas subrepresentadas como objeto de estudio, en particular, los casos de Cataluña y País Vasco. Destaca la representación mayoritaria de la Comunidad de Madrid y el núcleo central de dicha comunidad junto con las de Andalucía y Castilla-León. Así también, destaca el 
número de investigaciones que toman como muestra a estudiantes de las titulaciones de Ingeniería, mientras que las menos estudiadas son las de Artes y Humanidades. Sorprende que no haya más estudios en esta línea en el ámbito de las Ciencias de la Educación y que, de hecho, se trate de la disciplina que ocupa el quinto lugar -de un total de seis- en cuanto al volumen de estudios realizados en este ámbito. Teniendo en cuenta que el ámbito de la educación tendría que ser el pionero en el campo de la innovación, ya que es el que prepara a los futuros profesionales de la educación -los maestros y profesores-, no deja de ser alarmante que sea una de las disciplinas que menos investigaciones dedica al campo de la innovación a través de las tecnologías y, en este caso concreto, de los dispositivos móviles. Por lo tanto, este artículo se convierte en una invitación a los investigadores de universidades de las comunidades y disciplinas menos representadas para desarrollar experiencias e investigaciones en este ámbito, así como desarrollar estudios que acojan las áreas de conocimiento menos abordadas.

Enrelaciónalaspectometodológico,destacaladesproporciónentreinvestigaciones de carácter cuantitativo y cualitativo, siendo el primero ampliamente mayoritario. Esto puede explicarse por el hecho de que muchas publicaciones indexadas prioricen este tipo de investigación o que la aplicación de métodos estadísticos resulte más asequible para muchos investigadores. Esto resta, sin embargo, una mirada más compleja al tema de fondo, que va más allá de la caracterización del uso que se da a los dispositivos móviles y pasa por la aplicación en contextos específicos. En este sentido, es saludable el número cada vez mayor de sistematizaciones de experiencias realizadas en clase, aunque por la dimensión de muchos estudios, los resultados no sean extrapolables ni generalizables.

En suma, los hallazgos aquí presentados pueden constituir una línea de base sobre el estado de la investigación en dispositivos móviles en el contexto español, y dejan abierta una agenda por crecer en los años siguientes, especialmente si lo que se busca es elevar la eficiencia en los procesos de enseñanza y aprendizaje y la integración estratégica de tecnologías en el entorno de la educación superior.

\section{APOYOS}

Este estudio forma parte del proyecto $\mathrm{I}+\mathrm{D}+\mathrm{i}$ "Competencias mediáticas de la ciudadanía en medios digitales emergentes en entornos universitarios". EDU201564015-C3-2-R, financiado por el Ministerio de Economía y Competitividad de España. 


\section{REFERENCIAS BIBLIOGRÁFICAS}

Berge, Z., y Muilenburg, L. (Eds.). (2013). Handbook of Mobile Learning. Nueva York: Routledge.

Brazuelo, F., y Gallego, D. J. (2014). Estado del Mobile Learning en España. Educar Em Revista, 4, 99-128. doi: https://doi. org/10.1590/0104-4060.38646

Castañeda, L., y Adell, J. (2013). La anatomía de los PLEs. En L. Castañeda y J. Adell (Eds.), Entornos Personales de Aprendizaje: Claves para el ecosistema educativo en red (11-27). Alcoy: Marfil.

Delgado, E., y Repiso, R. (2013). Comunicar, 21(41), 45-52. doi: https://doi. org/10.3916/C41-2013-04

Durall, E., Gros, B., Maina, M., Johnson, L., y Adams, S. (2012). Perspectivas tecnológicas: educación superior en Iberoamérica 2012-2017. Austin, Texas: The New Media Consortium.

Fundación Telefónica (2011). Universidad 2020: Papel de las TIC en el nuevo entorno socioeconómico. Barcelona: Ariel.

ONTSI (2016). La Sociedad en Red. Informe Anual 2015 (Edición 2016). Madrid: Ministerio de Industria, Energía y Turismo.

Pimmer, C., Mateescu, M., y Gröhbiel, U. (2016). Mobile and Ubiquitous Learning in Higher Education Settings. A Systematic Review of Empirical Studies.
Computers in Human Behavior, 63, 490501. doi: http://dx.doi.org/10.1016/j. chb.2016.05.057

Píriz, S. (Ed.) (2015). UNIVERSITIC 2015. Análisis de las TIC en las Universidades Españolas. Madrid: Crue Universidades Españolas.

Pisanty, A. (2009) Presentación. Web 2.0: dispositivos móviles y abiertos para el aprendizaje. RIED. Revista Iboeroamericana de Educación a Distancia, 12(2). doi: http://dx.doi. org/10.5944/ried.2.12.898

Siemens, G. (2005). Connectivism: A Learning Theory for the Digital Age. International Journal of Instructional Technology and Distance Learning, 2(1), 3-10.

Sung, Y. T., Chang, K. E., y Liu, T. C. (2016). The effects of integrating mobile devices with teaching and learning on students' learning performance: A meta-analysis and research synthesis. Computers \& Education, 94, 252-275. doi:http://dx.doi. org/10.1016/j.compedu.2015.11.008

Villalonga, C., Marta-Lazo, D. C., y Es, C. (2015). Modelo de Integración Educomunicativa de “Apps” Móviles para la enseñanza y aprendizaje. PíxelBit. Revista de Medios y Educación, 46, 137-153 doi: https://doi.org/10.12795/ pixelbit.2015.i46.09

\section{PERFIL ACADÉMICO Y PROFESIONAL DE LOS AUTORES}

Julio César Mateus. Doctorando en Comunicación de la Universitat Pompeu Fabra, donde obtuvo el grado de Máster en Estudios Avanzados en Comunicación Social. Becario del Departamento de Comunicación y miembro del grupo de investigación MEDIUM (UPF). Profesor de la Universidad de Lima y la Pontificia Universidad Católica del Perú. Desarrolla su tesis doctoral sobre la educación mediática en la formación docente.

E-mail: julio.mateus@upf.edu 
María-José Masanet. Profesora en la Universitat Pompeu Fabra. Especialista en educación mediática, adolescencia, series de ficción y representación de la relación amorosa y de la sexualidad en los medios. Ha impartido seminarios sobre estos temas en diferentes universidades y ha publicado el libro "La educación mediática en la universidad española" (Gedisa, 2015) con el Dr. Joan Ferrés. Ha realizado estancias de investigación en la Loughborough University y en la Université Sorbonne Nouvelle, entre otras.

E-mail: mjose.masanet@upf.edu

\section{DIRECCIÓN DE LOS AUTORES}

Departamento de Comunicación

Universidad Pompeu Fabra

C/ Roc Boronat, 138

Barcelona (España)

Sue Aran-Ramspott. Profesora titular de Comunicación Audiovisual de la Universidad Ramon Llull. Premio de investigación en Comunicación (CAC, 2009). Consejera asesora en la CCMA, Corporación Catalana de Medios Audiovisuales. Miembro de DIGILAB, Strategy, Media and Regulation (URL). Especialista en Media Literacy, investiga sobre recepción; ficción audiovisual y representaciones de la violencia y de los estereotipos amorosos, y sobre los valores aspiracionales de los menores en Youtube.

E-mail: suear@blanquerna.url.edu

\section{DIRECCIÓN DE LA AUTORA}

Facultad de Comunicación y Relaciones Internacionales Blanquerna

Universidad Ramon Llull

Plaza Joan Corominas, $\mathrm{s} / \mathrm{n}$

Barcelona (España)

Fecha de recepción del artículo: 14/12/2016

Fecha de aceptación del artículo: 27/01/2017

\section{Como citar este artículo:}

Mateus, J. C., Aran-Ramspott, S., y Masanet, M. J. (2017). Revisión de la literatura sobre dispositivos móviles en la universidad española. RIED. Revista Iberoamericana de Educación a Distancia, 20(2), pp. 49-72. doi: http://dx.doi. org/10.5944/ried.20.2.17710 


\section{ANEXO: Artículos que conforman el corpus de estudio}

Agudo, J. E., Hernández-Linares, R., Rico, M., y Sánchez, H. (2014). Autoevaluación de la asistencia y la participación en clase con una Tablet PC. Revista Complutense de Educación, 25(2), 313-333. doi: http://doi.org/10.5209/ rev RCED.2014.v25.n2.41533

Ballester, E., Monsoriu, J. A., Castro-Palacio, J. C., Velázquez-Abad, L., Giménez, M. H., Monsoriu, J. A., y Sánchez Ruiz, L. M. (2014). Smart physics with smartphone sensors. IEEE Frontiers in Education Conference (FIE), 1-4. doi: https://doi. org/10.1109/FIE.2014.7044031

Berjón, R., Beato, M. E., Mateos, M., y Fermoso, A. (2015). Using Emerging Mobile Technologies to Enhance Collaborative Learning. A Study Case in a University Environment. International Journal of Education and Information Technologies, 9, 151-158.

Brazuelo, F., y Gallego, D. J. (2014). Estado del Mobile Learning en España. Educar Em Revista, 4, 99-128. doi: https://doi.org/10.1590/0104-4060.38646

Briz-Ponce, L., Juanes, J. A., y García-Peñalvo, F. J. (2015). Dispositivos móviles y apps: Características y uso actual en educación médica. Novática. Revista de la Asociación de Técnicos en Informática, 231, 86-91.

Briz-Ponce, L., y García-Peñalvo, F. J. (2015). An Empirical Assessment of a Technology Acceptance Model for Apps in Medical Education. Journal of Medical Systems, 39(11), 176. doi: https://doi.org/10.1007/s10916-015-0352-x

Bueno-García, J.-J., Colmenarejo-García, B., López-Rodríguez, M. C., y MartínezMaire, N. (2016). Tablets para el acceso a recursos electrónicos y apoyo a la docencia desde la biblioteca universitaria: un caso práctico. El Profesional de La Información, 25(6), 1699-2407. doi: https://doi.org/10.3145/epi.2016.nov.11

Camacho, M., y Tur, G. (2012). Exploring Learners' Practices and Perceptions on the Use of Mobile Portfolios as Methodological Tool to Assess Learning in Both Formal and Informal Contexts. 4th World Conference on Educational Sciences (WCES-2012), 46, 3182-3186 doi: https://doi.org/10.1016/j.sbspro.2012.06.03

Cantillo, C., Roura, M., y Sánchez, A. (2012). Tendencias actuales en el uso de dispositivos móviles en educación. La Educación Digital, 47, 1-21.

Carbonell, C., y Bermejo, L. A. (2016). Augmented Reality as a Digital Teaching Environment to Develop Spatial Thinking. Cartography and Geographic Information Science, o(o), 1-12. doi: https://doi.org/10.1080/15230406.2016.1145 556

Casanova, G., y Molina, J. M. (2013). Implementación de códigos QR en materiales docentes. XI Jornadas de redes de investigación en docencia universitaria, 53, 1689-1699. doi: https://doi.org/10.1017/CBO9781107415324.004

Del Campo, E. (2013). M-Learning y aprendizaje informal en la educación superior mediante dispositivos móviles. Historia y Comunicación Social, 18, 231242. doi: http://dx.doi.org/10.5209/rev HICS.2013.v18.44239 
Fombona, J., Pascual, M. A., y Ferreira Amador, M. F. (2012). Realidad aumentada, una evolución de las aplicaciones de los dispositivos móviles. Píxel-Bit. Revista de Medios y Educación, 41, 197-210.

Fombona, J., y Pascual, M. A. (2013). Beneficios del m-learning en la Educación Superior. Educatio Siglo XXI, 31(2), 211-234.

Galindo-Rubio, F., Suárez-Carballo, F., y Martín, J. R. (2016). Análisis de la eficacia de una metodología didáctica basada en el uso de smartphones con estudiantes de comunicación. Prisma Social, 15, 1-33.

Gallardo, E., Marqués, L., y Bullen, M. (2015). El estudiante en la educación superior: Usos académicos y sociales de la tecnología digital. RUSC. Universities and Knowledge Society Journal, 12(1), 25-37. doi: https://doi.org/10.7238/rusc. v12i1.2078

Gallardo, J. (2013). Análisis de la integración del iPad en el aula desde la perspectiva del alumno: proyecto piloto de la UCJC. Historia y Comunicación Social, 18, 399-410. doi: http://dx.doi.org/10.5209/rev HICS.2013.v18.43975

González-Fernández, N., y Salcines, I. (2015). El teléfono inteligente en los procesos de enseñanza-aprendizaje-evaluación en Educación Superior. Percepciones de docentes y estudiantes. RELIEVE, 21(2), 1-20.

González-Fernández, N., y Salcines, I. (2015). Los smartphones en Educación Superior. Diseño y Validación de dos instrumentos de recogida de información sobre la visión del alumnado. REOP. Revista Española de Orientación y Psicopedagogía, 26(3), 96-120.

Gros, B., y Noguera, I. (2013). Mirando el futuro: Evolución de las tendencias tecnopedagógicas en Educación Superior. Campus Virtuales. Revista Científica de Tecnología Educativa, 2(2), 130-140.

Guerrero, C., Jaume, A., Juiz, C., y Lera, I. (2016). Use of mobile devices in the classroom to increase motivation and participation of engineering university students. IEEE Latin America Transactions, 14(1), 411-416. doi: https://doi. org/10.1109/TLA.2016.7430109

Hernández, C., Vegas, J., Llamas, C., \& González, M. A. (2014). A survey on mobile devices use by university students. International Symposium on Computers in Education (SIIE 2014), 223-226.

Humanante-Ramos, P., García-Peñalvo, F. J., y Conde-González, M. Á. (2016). PLEs in mobile contexts: new ways to personalize learning. IEEE VAEP-RITA, 4(1), 33-39.

Laborda, J. G., Royo, T. M., Lazaro, N. R., y Marugan, L. F. (2015). Facilitating Language Tests Delivery Through Tablet PC's. 7th World Conference on Educational Sciences, 197 (February), 1302-1306. doi: https://doi.org/10.1016/j. sbspro.2015.07.403

López Hernández, F. A., y Silva, M. M. (2016). Factors of Mobile Learning Acceptance in Higher Education. Estudios sobre Educación, 30, 175-195. doi: https://doi.org/10.15581/004.30.175-195 
Marcelo, C., Yot, C., y Mayor, C. (2015). Enseñar con tecnologías digitales en la Universidad. Comunicar, 23(45), 117-124. doi: https://doi.org/10.3916/C45-2015$\underline{12}$

Martín, N., Sánchez, I., Bravo, M., Hernández, J., Saorin, J. L., y Contero, M. (2014). Virtual Blocks: a serious game for spatial ability improvement on mobile devices. Multimedia Tools and Applications, 73(3), 1575-1595. doi: https://doi. org/10.1007/s11042-013-1652-0

Martínez-Solana, M. (2014). Redes sociales y TIC, su papel en la educación superior del siglo XXI. Historia y Comunicación Social, 19, 63-71. doi: https://doi. org/10.5209/rev_HICS.2014.v19.45108

Menchaca, I., Guenaga, M., y Romero, S. (2015). Mobile devices, powerful teaching tools in the engineering classroom. IEEE Global Engineering Education Conference, EDUCON2O15, 577-581. doi: https://doi.org/10.1109/EDUCON.2015.7096027

Millán, A., y Pérez Manzano, A. (2014). Usabilidad de tablets para el acceso a campus virtuales universitarios por alumnos con discapacidad. Historia y Comunicación Social, 19(Esp. Febrero), 805-818. doi: https://doi.org/10.5209/ rev HICS.2014.v19.45067

Millor, M., Etxano, J., Slon, P., García-Barquín, P., Villanueva, A., Bastarrika, G., y Pueyo, J. C. (2015). Use of remote response devices: an effective interactive method in the long- term learning. European Radiology, 25(3), 894-900. doi: https://doi. org/10.1007/s00330-014-3468-3

Molina, H. (2013). La educación universitaria en el bolsillo, aplicaciones y entornos virtuales. Estudios sobre el mensaje periodístico, 19, 319-329. doi: https:// doi.org/10.5209/rev ESMP.2013.v19.42039

Molina, P., Valenciano, J., y Valencia-Peris, A. (2015). Los blogs como entornos virtuales de enseñanza y aprendizaje en Educación Superior. Revista Complutense de Educación, 26, 15-31. doi: https://doi.org/10.5209/rev RCED.2015.v26.43791

Pascual, M. A. (2013). La universidad ante las posibilidades de los dispositivos móviles en el aprendizaje ubicuo. Historia y Comunicación Social, 18, 461-468. doi: http://dx.doi.org/10.5209/rev HICS.2013.v18.44255

Peña, F. D., Burgos, C., y Simón, M. A. (2015). Mobile learning multidispositivo en la enseñanza universitaria mediante el uso de aplicaciones Web. Experiencia en la asignatura Dirección de la Producción. Educade: Revista de Educación en Contabilidad, Finanzas y Administración de Empresas, 6, 59-79.

Pinto, M., Pouliot, C., y Cordón-García, J. A. (2014). E-book reading among Spanish university students. The Electronic Library, 32(4), 473-492. doi: http:// dx.doi.org/10.1108/EL-05-2012-0048

Quicios, M. I., Sevillano, M. L., y Ortega, I. (2013). Educational uses of mobile phones by university students in Spain. New Educational Review, 34(4), 151-163.

Rius, À., Masip, D., y Clarisó, R. (2014). Proyectos delos estudiantes para potenciar el aprendizaje móvil en la educación superior. Aplicaciones para el aprendizaje móvil en educación superior. Revista de Universidad y Sociedad del Conocimiento (RUSC), 11(1), 193-207. doi: http://dx.doi.org/10.7238/rusc.v11i1.1901 
Santamarta, J. C., Hernández-Gutiérrez, L. E., Tomás, R., Cano, M., RodríguezMartín, J., y Arraiza, M. P. (2015). Use of Tablet Pcs in Higher Education: A new Strategy for Training Engineers in European Bachelors and Masters Programmes. Proceedings of 6th World Conference on Educational Sciences, 191, 2753-2757. doi: https://doi.org/10.1016/j.sbspro.2015.04.657

Saorin, J. L., Torre, J. de La, Martín, N., y Carbonell, C. (2013). Spatial Training using Digital Tablets. 3 rd World Conference on Learning, Teaching and Educational Leadership, 93, 1593-1597. doi: https://doi.org/10.1016/j.sbspro.2013.10.087

Sevillano, M. L., Quicios-García, M. P., y González-García, J. L. (2016). Posibilidades ubicuas del ordenador portátil: percepción de estudiantes universitarios españoles. Comunicar, 24(46), 87-95. doi: http://doi.org/10.3916/C46-2016-09

Sevillano, M. L., y Vázquez, E. (2014). Análisis de la funcionalidad didáctica de las tabletas digitales en el Espacio Europeo de Educación Superior. Revista de Universidad y Sociedad del Conocimiento (RUSC), 11(3), 47-81. doi: https://doi. org/10.7238/rusc.v11i3.1808

Sevillano, M. L., y Vázquez, E. (2015). The Impact of Digital Mobile Devices in Higher Education. Educational Technology y Society, 18(1), 106-118.

Tur, G., Marín, V. I., y Marín, V. I. (2014). Enhancing learning with the social media: student teachers' perceptions on Twitter in a debate activity. Journal of New Approaches in Educational Research, 4(1), 46-43. doi: https://doi.org/10.7821/ naer.2015.1.102

Vázquez, E. (2014). Mobile Distance Learning with Smartphones and Apps in Higher Education. Educational Sciences: Theory y Practice, 14(4), 1505-1520. doi: https://doi.org/10.12738/estp.2014.4.2012

Vázquez, E. (2015). El reto de la formación docente para el uso de dispositivos digitales móviles en la educación superior. Perspectiva Educacional, 54(1), 149-162. doi: https://doi.org/10.4151/07189729-Vol.54-Iss.1-Art.236

Vázquez, E., Sevillano, M. L., y Fombona, J. (2016). Análisis del uso educativo y social de los dispositivos digitales en el contexto universitario panhispánico. Revista de Investigación Educativa, 34(2), 453-469. doi: https://doi.org/10.6018/ rie.34.2.224691

Vázquez, E., y Sevillano, M. L. (2015). El smartphone en la educación superior. Un estudio comparativo del uso educativo, social y ubicuo en universidades españolas e hispanoamericanas. Signo y Pensamiento, 34(67), 132-149. doi: https:// doi.org/10.11144/Javeriana.syp34-67.sese

Vílchez, M. J., Reche, E., y Marín, V. (2015). Diseño y validación de un cuestionario para valorar whatsapp en la regulación de trabajo en grupo. Etic@net, 2(15), 245-272.

Villalonga, C., Marta-Lazo, D. C., y Es, C. (2015). Modelo de Integración Educomunicativa de "Apps" Móviles para la enseñanza y aprendizaje. PíxelBit. Revista de Medios y Educación, 46, 137-153. doi: https://doi.org/10.12795/ pixelbit.2015.i46.09. 